\section{Triple wavelength LED driver for optical brain-computer interfaces}

\section{C.J. Soraghan, C. Markham, F. Matthews and T.E. Ward}

A dedicated triple wavelength LED driver is presented for optical brain-computer interfacing (BCI). The solution caters for the constraints of a common-anode grounded case and modulation up to several kilohertz that allows source separation of light that has backscattered from the brain. With total harmonic distortion of $0.95 \%$ and a frequency range of $\sim 40 \mathrm{kHz}$, the driver has application in a continuous wave optical BCI. Other modulation strategies such as time division multiplexing (TDM) are catered for, owing to input DC coupling. Linearity in the optical output is maintained by the 'load sensing' differential op-amp on the LED's current limiting resistor, which is the basis for the $V-I$ conversion.

Introduction: Continuous wave near-infrared spectroscopy (CWNIRS) is now an established imaging modality for assessing brain state. In particular, it has specialised applications in brain-computer interfaces (BCI) [1] and bedside monitoring for neonates [2], with further potential for use in stroke rehabilitation [3]. The light emitting diode (LED) driver described here was constructed for dedicated use in an optical braincomputer interface (OBCI).

A BCI is a device which provides a user with an alternative communication channel independent of the normal output pathways of the brain (the spinal cord and peripheral nervous system). Currently the optical method used to assess functional activity directly from the user's brain for use in a BCI is CWNIRS. In this method non-ionising nearinfrared (NIR) light is shone onto the subject's head, a suitable detector placed a few centimetres away from the light source collects the backscattered photons. Changes in concentration levels of oxyhaemoglobin $(\mathrm{HbO})$ and deoxy haemoglobin (HbR) in cortical tissue can be calculated from the levels of absorption and scattering of the light detected. These haemoglobin concentration changes report on the intentional functional activity by the user and can be exploited for communication in a BCI. In order to obtain concentration changes in both $\mathrm{HbO}$ and $\mathrm{HbR}$, at least two wavelengths of light are required (see [4] for more on this technique).

Motivation: The optical BCI requires a circuit to control the NIR light sources. The light sources chosen were high power NIR LEDs which have features suitable for long term use in a BCI. The use of LEDs in an offline optical BCI for ALS patients [5] and for imaging neonates [2] has been demonstrated successfully by others in the literature, verifying LED applicability and indeed safety in human interfacing. In addition, a study by the authors of the safety aspects of the LEDs described here can be found in [6]. To retrieve $\mathrm{HbO}$ and $\mathrm{HbR}$ concentration changes, the optical $\mathrm{BCI}$ therefore requires multiple NIR light sources.

LEDs are current driven devices. The current through them is directly proportional to the optical output power they deliver. Preferably, these devices should be voltage controlled. Thus, a stable voltage-to-current converter, which essentially provides voltage-to-light intensity (linear) conversion, is required. A further requirement is that for CWNIRS, the driver must allow for modulation of the light output from DC to several kilohertz. In addition input DC coupling is required to facilitate multiple modulation strategies such as time division multiplexing (TDM).

To ensure maximum light entry into the head at a focused point on the scalp, all three wavelengths (the third wavelength was chosen to give an independent measure of total-haemoglobin $(\mathrm{HbT})$ measurable at the isosbestic point $(800 \mathrm{~nm})$, as well as $\mathrm{HbR}$ and $\mathrm{HbO})$ should be housed within the same LED package. Without common anode/cathode configuration, this would require at least six leads from the device, making the resulting system bulky. Thus the custom-made commonanode (ground) triple wavelength LEDs used (APT0101- Opto Diode Corp.) place an extra constraint on the LED driver design. The common-anode connection must be grounded. This favourable common-ground configuration also has a potential safety benefit, since the LED package case is also connected to ground. Indeed, it allows shielding right up to the active component (LED dye). Statistically the popular design in Fig. 1 has the potential for lower inherent noise, but nevertheless it cannot satisfy the common-anode ground constraints, which are satisfied by the driver design presented here (Fig. 2).

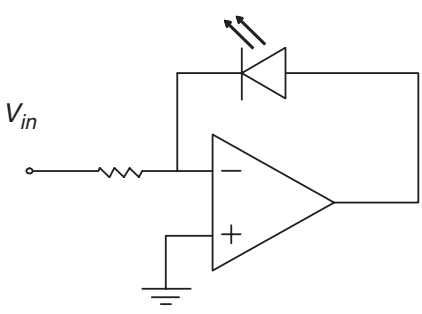

Fig. 1 Standard active V-I converter design

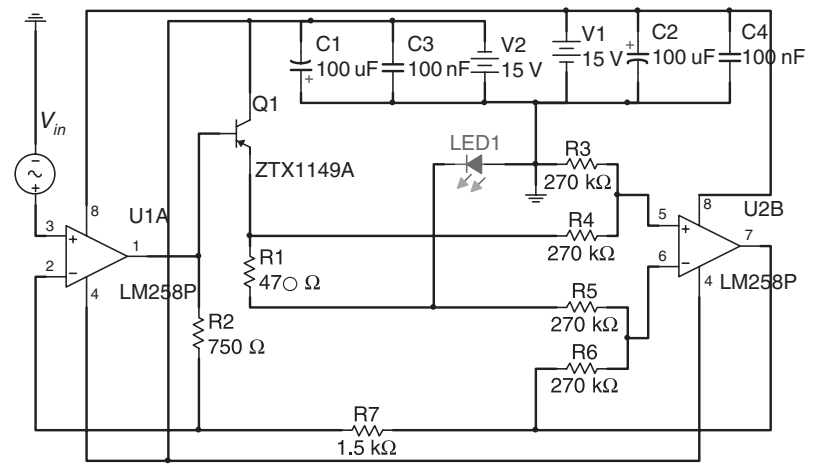

Fig. 2 LED driver subcircuit schematic (three subcircuits per APT0101 package)

Each subcircuit in schematic has same components, only one is shown for convenience. The input voltage and LED are shown in subcircuit to outline a single dye's complete circuitry

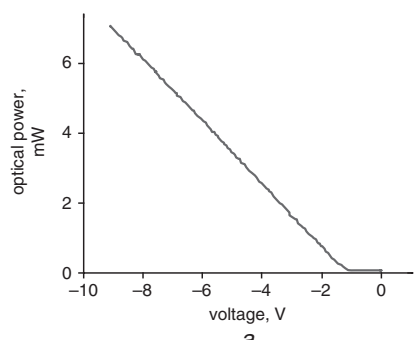

a

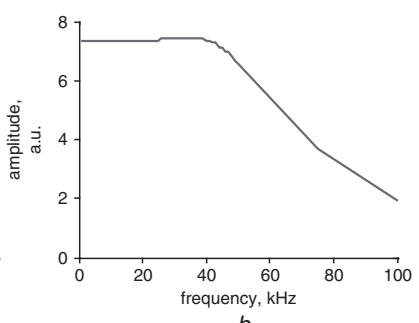

$b$

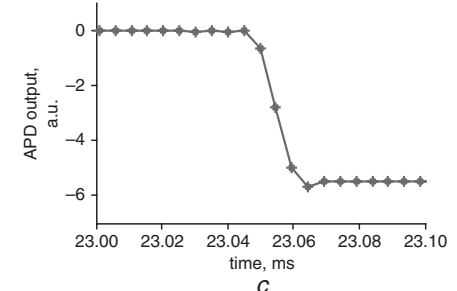

Fig. 3 LED driver characteristics

$a$ Optical power output linear response to change in DC input voltage (slope $=$ $-0.89950 .0006[\mathrm{~mW} / V])$; (intercept $=-1.0634 \pm 0.0027[V])$

$b$ Frequency response of LED driver ( $\sim 40 \mathrm{kHz}$ range);

$c$ Step response of LED driver.

Circuit description: The necessary modifications from the standard active driver design (Fig. 1) are $p n p$ transistors for amplification, a differential op-amp, resistors to reduce the gain in the second stage (required to suppress oscillations in the output), and capacitors to decouple the supply rails.

Each dye (single wavelength in the LED triple wavelength package) requires a maximum $\sim 140 \mathrm{~mA}$ RMS driving current. Op-amp rails were held at $\pm 15 \mathrm{~V}$. Typical input voltage levels $\left(V_{\text {in }}\right)$ were $-5.67 \mathrm{VDC}$ and 2.35 VAC-RMS. LED driver AC frequencies are set in the range 1$20 \mathrm{kHz}$ (for separation of the multiple light sources at the detector end).

The differential op-amp stage facilitates grounding of the commonanode leads. This op-amp feeds back the potential drop across the LED's current limiting resistor $(1 \mathrm{~W}, 47 \Omega)$ in order to sense the current driving the LED. This reduces the error (approximating linear mapping) in the voltage-to-light intensity output by each LED and is the basis for the $V-I$ conversion stability. Oscillations $(\sim 0.333 \mathrm{MHz})$ in the output were observed in the initial Electronics Workbench (Multisim) simulation of the circuit. These were removed by reducing 
the feedback gain in each subcircuit. The feedback resistors (R2 and R7) resolved this issue in the simulation and in practice.

Results: Fig. $3 a$ demonstrates the voltage-to-light intensity mapping (using an optical power meter (Model: Ando AQ-2105)) showing the desired linearity. The offset (non-zero intercept) in the Figure is due to the potential drop by the feedback resistors R2 and R7 (required to reduce the aforementioned oscillations). This offset is catered for by maintaining a DC offset at the voltage input, to ensure the LED is always on. The frequency response $(\sim 40 \mathrm{kHz}$ range) and step response (sampled at $204.8 \mathrm{kHz}$ ) are shown in Fig. $3 b$ and Fig. $3 c$, respectively. Finally, total harmonic distortion of $0.95 \%$ was recorded at the detector output, using a LabVIEW virtual instrument.

Conclusion: The LED driver described caters for scaling an OBCI to a multi-channel device which is needed to improve localisation and throughput/data rates of the BCI device. Since the input design is DC coupled it also enables the researchers to test and establish different modulation strategies such as time division multiplexing, making the device more flexible/universal for different research needs. It should also be noted that the LEDs require about $35 \mathrm{~min}$ settling time before use so as to stabilise their temperature and thus the drift in the optical output [6]. Care should also be taken to avoid clipping (this introduces harmonics and thus poor demodulation). With a frequency range of $\sim 40 \mathrm{kHz}$ the LED driver is suitable for CWNIRS [7].

Acknowledgments: The authors acknowledge the contributions from the late W. Lanigan from the Department of Experimental Physics. The authors also thank P. Serry and G. Barrett for their comments and suggestions. This work is supported by Science Foundation Ireland (SFI) under grant number SFI/05/RFP/ENG0089.

C) The Institution of Engineering and Technology 2009

22 January 2009

doi: $10.1049 / \mathrm{el} .2009 .0214$
C.J. Soraghan (Department of Experimental Physics c/o Department of Computer Science, National University of Ireland Maynooth, County Kildare, Ireland)

E-mail: christopher.j.soraghan@nuim.ie

C. Markham (Department of Computer Science, National University of Ireland Maynooth, County Kildare, Ireland)

F. Matthews (Hamilton Institute c/o Department of Computer Science, National University of Ireland Maynooth, County Kildare, Ireland)

T.E. Ward (Department of Electronic Engineering, National University of Ireland Maynooth, County Kildare, Ireland)

\section{References}

1 Coyle, S., Ward, T., Markham, C., and McDarby, G.: 'On the suitability of near-infrared (NIR) systems for next-generation brain-computer interfaces', Physiol. Meas., 2004, 25, (4), pp. 815-22

2 Bozkurt, A., Rosen, A., Rosen, H., and Onaral, B.: 'A portable near infrared spectroscopy system for bedside monitoring of newborn baby', Biomed. Eng. Online, 2005, 4, (1), p. 29

3 Ward, T.E., Soraghan, C.J., Matthews, F., and Markham, C.: 'A concept for extending the applicability of constraint-induced movement therapy through motor cortex activity feedback using a neural prosthesis', Comput. Intell. Neuroscience, 2007, 2007, (9), Article ID 51363

4 Matthews, F., Pearlmutter, B.A., Ward, T.E., Soraghan, C., and Markham, C.: 'Hemodynamics for brain-computer interfaces', IEEE Signal Process. Mag., 2008, 25, (1), pp. 87-94

5 Naito, M., Michioka, Y., Ozawa, K., Ito, Y., Kiguchi, M., and Kanazawa, T.: 'A communication means for totally locked-in ALS patients based on changes in cerebral blood volume measured with near-infrared light', IEICE Trans. Inf. Syst. (Japan), 2007, E90d, (7), pp. 1028-1037

6 Soraghan, C., Ward, T.E., Matthews, F., and Markham, C.: 'Optical safety assessment of a near-infrared brain-computer interface'. Proc. IEE Irish Signals and Systems Conf., Galway, Ireland, June 2008

7 Soraghan, C., Matthews, F., Markham, C., Pearlmutter, B.A., O’Neill, R., and Ward, T.E.: 'A 12-channel, real-time near-infrared spectroscopy instrument for brain-computer interface applications'. Proc. 30th Annual International Conf. of IEEE Engineering in Medicine and Biology Society, Vancouver, BA, Canada, August 2008 